\title{
Compound action potentials recorded in the ventral tegmental area, substantia nigra, and periaqueductal gray following rewarding stimulation of the lateral hypothalamus in the rat
}

\author{
IVAN KISS and PETER SHIZGAL \\ Concordia University, Montreal, Quebec, Canada
}

\begin{abstract}
Neurons activated by rewarding stimulation of the lateral hypothalamus (LH) were studied by two complementary means. To ensure that the same directly stimulated neurons were activated in both tests, the same subjects, stimulation sites, currents, and pulse durations were employed. In the first test, Yeomans's method was used to obtain behaviorally derived measures of recovery from refractoriness in the neurons responsible for the rewarding effect. In the second test, electrophysiological recording was used to measure recovery from refractoriness in the neurons responsible for compound action potentials (CAPs) evoked by the rewarding stimulation. Refractory-period estimates derived from CAPs recorded in the ventral tegmental area (VTA), substantia nigra (SN), and periaqueductal gray matter (PAG) were found to overlap the refractoryperiod estimates for the directly stimulated neurons subserving the rewarding effect. These findings reinforce the view that reward-related fibers directly link the LH and VTA, and suggest that fibers similar to those responsible for the rewarding effect link the LH to the SN and PAG.
\end{abstract}

Evidence obtained by means of a behavioral adaptation of the collision test (Bielajew \& Shizgal, 1982; Shizgal, Bielajew, Corbett, Skelton, \& Yeomans, 1980) suggests that at least some of the directly activated neurons responsible for the rewarding effect of stimulating the medial forebrain bundle (MFB) have relatively long axons that directly link the lateral hypothalamus (LH) and ventral tegmental area (VTA). Behaviorally derived estimates of the refractory periods of these neurons often fall within 0.4-1.5 msec (Shizgal \& Murray, 1989; Yeomans, 1975), although a contribution of more slowly recovering fibers can be discerned if high currents and small electrode tips are used (Yeomans, Mercouris, \& Ellard, 1985). If the inferences drawn from the behavioral data are correct, then rewarding stimulation of the LH should trigger a volley that could be registered as a compound action potential (CAP) at the VTA. Furthermore, the refractory periods of the neurons responsible for the CAPs should overlap with behaviorally derived estimates for MFB reward neurons.

This work was supported by a grant to Peter Shizgal from the Medical Research Council of Canada (MA-8037) and by a grant from the Fonds FCAR du Québec (CE-103) to the Centre for Studies in Behavioural Neurobiology (R. A. Wise, principal investigator). The authors thank Margaret Forgie, Kent Conover, Beverley Murray, and Dwayne Schindler for their helpful comments on the manuscript, and Kelly Rothney for her assistance with the figures. Ivan Kiss's present address is Edmonton General Hospital, Department of Psychology, 11111 Jasper Avenue, Edmonton, Alberta T5K 0L4, Canada. Correspondence may be addressed to P. Shizgal, Centre for Studies in Behavioural Neurobiology, Concordia University, 1455 de Maisonneuve Blvd. W., Montreal, Quebec H3G 1M8, Canada.
The results of an earlier CAP recording study (Shizgal, Bielajew, \& Kiss, 1982) are consistent with these predictions: shortly following stimulation of the $\mathrm{LH}$, we recorded CAPs in the VTA. When pairs of pulses were used to drive the CAPs, the response to the second pulse appeared to diminish as the intrapair interval was decreased to values within the range of behaviorally derived estimates of the refractory periods of MFB reward neurons. However, the onset of recovery from refractoriness was often difficult to discern because the response to the second pulse ( $\mathrm{T}$ pulse) was superimposed on components of the shock artifact(s) and the response to the first pulse (C pulse). Furthermore, the stimulation sites were neither screened for self-stimulation nor used to obtain behaviorally derived estimates of the refractory periods of the neurons responsible for the rewarding effect. Thus, we could not be certain that the stimulation driving the CAPs would have been rewarding, and we had to compare the refractory-period estimates derived from the CAPs to values obtained with different stimulation electrodes and currents.

We have recently developed an improved method for rejecting shock artifacts from CAP records and for isolating T-pulse responses (Kiss \& Shizgal, 1989). In the work reported here, these methods were applied to more clearly describe the CAPs elicited by rewarding stimulation of the LH. To ensure that the stimulation was rewarding, only electrodes that supported vigorous self-stimulation were used to drive the CAPs. To ensure that the directly stimulated neurons were essentially the same in the behavioral and electrophysiological phases of the study, the same current and pulse duration were employed. These stimulation parameters were used to obtain behaviorally 
derived estimates of refractory periods prior to the recording session. Thus, variability due to differences in stimulation site and current was eliminated from the comparison of behaviorally and electrophysiologically derived estimates of refractory periods.

To reassess our initial characterization of the neurons responsible for the CAPs elicited by MFB stimulation, recording electrodes were aimed at the VTA. To extend the original findings, recording electrodes were also aimed at two other sites where self-stimulation has been observed-the substantia nigra (SN) and the periaqueductal gray matter (PAG). We reasoned that if CAPs elicited by rewarding stimulation of the $\mathrm{LH}$ were registered at the SN and PAG sites, and if the refractory periods of the fibers that give rise to these CAPs overlapped with the behaviorally derived estimates of the refractory periods of reward neurons activated by LH stimulation, then these sites could be regarded as promising targets for future experiments in which the behavioral version of the collision technique could be used to determine whether the fibers linking the $\mathrm{LH}$ to the recording sites include the axons of reward neurons.

\section{METHOD}

\section{Subjects}

Thirteen male Long-Evans rats (Canadian Breeding Farms) weighing between 330 and $500 \mathrm{~g}$ at the time of initial surgery served as subjects. They were maintained on a reverse 12 -h light/12-h dark cycle and were given ad lib access to Purina rat chow and water.

\section{Electrodes}

Stimulating electrodes were fashioned from stainless steel wires, $11 \mathrm{~mm}$ long and $0.25 \mathrm{~mm}$ in diameter. The tips were honed to a roughly hemispheric shape, and the shafts were insulated with enamel (Formvar).

Recording electrodes were composed of pairs of enamel-insulated stainless steel wires, $0.13 \mathrm{~mm}$ in diameter. The tips were square-cut; in some cases, the shafts were bared of insulation within $0.25 \mathrm{~mm}$ of the tips. The electrodes were glued together using epoxy or lacquer so that the tips were separated by about $0.5 \mathrm{~mm}$ along the long axis of the shafts.

\section{Initial Surgery}

Chronic stimulating electrodes were aimed bilaterally at the $\mathrm{LH}$ using standard stereotaxic methods (level skull coordinates: $2.8 \mathrm{~mm}$ posterior to bregma, $1.7 \mathrm{~mm}$ lateral to the midsagittal suture, and 7.7-7.8 $\mathrm{mm}$ below the dura mater). The reader is referred to an earlier publication (Kiss \& Shizgal, 1989) for details.

\section{Histology}

After completion of the recording sessions, the subject was perfused intracardially with $100 \mathrm{ml}$ of isotonic saline followed by $100 \mathrm{ml}$ of $10 \%$ formalin containing $3 \mathrm{~g}$ of potassium ferrocyanide, $3 \mathrm{~g}$ of potassium ferricyanide, and $0.5 \mathrm{~g}$ trichloracetic acid. This latter solution formed a blue reaction product at the sites of marking lesions made at the bottom of each penetration of the recording electrode and at the tip of the stimulation electrode. The brain was removed and stored in $10 \%$ formalin for at least 1 week. Frozen 20- or $40-\mu \mathrm{m}$ sections were mounted on gelatin-coated slides and stained using the formol-thionin method.

\section{Behavioral Methods}

Apparatus. The subjects were shaped to self-stimulate in chambers $70 \mathrm{~cm}$ high, $25 \mathrm{~cm}$ wide, and $25 \mathrm{~cm}$ deep, with wire-mesh floors. A rodent lever (Lehigh Valley) was mounted in the center of one wall, roughly $5 \mathrm{~cm}$ above the floor. About $5 \mathrm{~cm}$ above the lever was a yellow "jewel" light measuring $1.5 \mathrm{~cm}$ in diameter; this light was illuminated whenever stimulation was available and extinguished either during delivery of a stimulation train or between testing trials. The stimulation leads terminated in a graphite-on-gold slip-ring assembly (Airflyte Electronics CAY-652-7) that allowed the subject to circle, without twisting the leads, while maintaining electrical continuity.

The temporal parameters of the stimulation were controlled by integrated-circuit pulse generators, whereas the amplitude was set by dual-channel constant-current amplifiers (Mundl, 1980). Accumulation of charge at the electrode-brain interface was minimized by a circuit that shorted the stimulator outputs through a $1-\mathrm{k} \Omega$ resistor in the absence of a pulse on either channel. Current was monitored by observing the voltage drop across a $1 \%$ precision, $1-\mathrm{k} \Omega$ resistor in series with the rat.

With the following exceptions, the computer-controlled apparatus used to obtain refractory-period estimates resembled the manually controlled setup described above. The test chambers were built entirely of Plexiglas and had either solid floors covered with wood chips or floors fashioned from Plexiglas rods. The lever was mounted toward one corner of the chamber, about $5 \mathrm{~cm}$ from the wall. The test chambers were mounted in ventilated, soundattenuated plywood enclosures illuminated by a $40-\mathrm{W}$ bulb. Plexiglas panels in the plywood doors permitted the subjects' behavior to be monitored from an adjoining room by means of a video camera.

Temporal parameters of the stimulation for each test station were controlled by a dedicated M6800-based microcomputer (Motorola M68MM01D). A bank of relays connected to the parallel port of the microcomputer selected the stimulation electrodes, and the current was delivered by a dual voltage-controlled constant-current amplifier (Mundl, 1980) referenced by a digital-to-analog converter. Software running on a PDP-11/73 computer selected the stimulation parameters to be used and downloaded them into the microcomputers via a serial interface. At the end of each $30-\mathrm{sec}$ trial, the number of barpresses was reported to the PDP-11/73 by the microcomputer, and new parameters were selected.

Screening and shaping. Several days after surgery, the LH electrodes were tested to ascertain whether they would support selfstimulation. The subjects were shaped to press the lever to receive 0.5 -sec trains of 0.1 -msec cathodal rectangular pulses. The number of pulses per train and the current were adjusted to yield vigorous responding (at least $40 \mathrm{presses} / \mathrm{min}$ ) and to minimize concomitant stimulation-induced movements. The number of pulses was then manually decreased in $0.1 \log _{10}$-unit steps until responding ceased and the number of pulses required to support a half-maximal rate of responding (the "required number") computed by interpolation. This procedure for determining the required number was repeated until the estimates varied by less than $0.2 \log _{10}$ units. Subjects that showed pronounced, disruptive movements in response to the stimulation or failed to meet the above-mentioned rate and stability criteria were eliminated from the study.

Stabilization and estimation of refractory periods. These two phases of the experiment were carried out in the computer-controlled test chambers. The required number of pulses was estimated by means of the following algorithm. The number of pulses tested on the first trial of a determination was read from a user-supplied file. If the rat did not respond on the first trial of a determination, the number of pulses was increased in $0.3 \log _{10}$-unit steps until responding was elicited. Once the subject began to respond, the number of pulses was increased in $0.1 \log _{10}$-unit steps until the response 
rate failed to exceed the rate on the previous trial by $10 \%$. (A preset limit was defined to prevent the system from delivering an excessive number of pulses.) The number of pulses was then set to a value $0.1 \log _{10}$ units below the starting value and was decreased in $0.1 \log _{10}$-unit steps on successive trials until the response rate fell below $10 \%$ of the maximum rate for that determination. A final trial was then run after the number of pulses was again decremented by $0.1 \log _{10}$ units.

Stabilization sessions consisted of repeated determinations of the required number of pulses. Performance was considered stable when the required number varied by no more than $0.1 \log _{10}$ units during a session.

Sessions in which refractory periods were estimated consisted of two kinds of determinations of the required number. The first four determinations and every fifth determination thereafter were carried out using stimulation trains composed of single pulses, whereas the remaining determinations were carried out using trains composed of pulse pairs. A different $\mathrm{C}-\mathrm{T}$ interval ranging from $0.5-5.0 \mathrm{msec}$ was tested in each determination of the required number of pulse pairs.

The measure of recovery from refractoriness was based upon the change in the required number as a function of $\mathrm{C}-\mathrm{T}$ interval. The effectiveness of the $T$ pulse was estimated using the following formula (Yeomans, 1975):

$$
E=\frac{R N_{\mathrm{SP}}}{R N_{\mathrm{CT}}}-1,
$$

where $E=$ effectiveness of the $\mathrm{T}$ pulse, $R N_{\mathrm{SP}}=$ the average required number of single pulses, and $R N_{\mathrm{CT}}=$ the required number of pulse pairs at a given C-T interval. (The first two determinations of the required number of single pulses were considered "warm-ups" and were not included in the calculations.)

In principle, this formula yields a value of zero when the $T$ pulse fires no reward neurons. When the $T$ pulse fires the same number of reward neurons as the $C$ puise, $E$ is equal to 1 .

\section{Electrophysiological Methods}

The surgical procedures and recording apparatus are described in detail elsewhere (Kiss \& Shizgal, 1989), and hence are only briefly summarized here.

The recording session was carried out under deep urethane (ethyl carbamate, $1.2 \mathrm{~g} / \mathrm{kg}$ ) anesthesia, after completion of the behavioral testing. Recording electrodes were composed of pairs of enamelinsulated stainless steel wires, $0.13 \mathrm{~mm}$ in diameter, with squarecut tips. In some cases, the shafts were bared of insulation within $0.25 \mathrm{~mm}$ of the tips. The electrodes were glued together using epoxy or lacquer so that the tips were separated by $0.5 \mathrm{~mm}$ along the long axis of the shafts.

Signals from the two poles of the recording electrodes were separately amplified and filtered before being combined in a differential amplifier and stored on FM tape. Averaging and digital subtraction were carried out off-line using a microcomputer.

The stimulation used to drive the CAPs was delivered by a pair of constant-current amplifiers (Grass CCIU1) connected to a custombuilt pulse generator by isolation units (Grass SIU5). Stimulation pulses of the same current, polarity, and pulse duration as used in the behavioral phase of the experiment were delivered via the same LH stimulation electrode.

The tip of the recording electrode was positioned within a region bounded by the following coordinates: $0-3.0 \mathrm{~mm}$ lateral to the midsagittal suture, 4.8-8.3 mm posterior to bregma, and 4.0-10.5 mm below the dura mater. In an attempt to elicit CAPs, stimulation was delivered via the LH electrode at a frequency of 1-2 Hz. If no CAP was observed at a given position, the recording electrode was lowered $0.5 \mathrm{~mm}$. Once a putative CAP was observed, the stimulation frequency was increased to $10,50,100$, and $300 \mathrm{~Hz}$ and any changes in the CAP were noted. Recovery from refractoriness in the neurons responsible for the $\mathrm{CAP}$ was then measured as described below. Following the refractory-period measurements, the recording electrode was again lowered $0.5 \mathrm{~mm}$. If a putative CAP was observed, the frequency test and refractory-period measurements were repeated. If no CAP was observed, the recording electrode was again lowered $0.5 \mathrm{~mm}$. This process was repeated until the computed position of the recording electrode approached the bottom of the brain.

Measurement of refractory periods. To measure recovery from refractoriness in the neurons responsible for the CAPs, pairs of stimulation pulses were delivered and the $C-T$ interval was varied. To improve artifact rejection and isolation of T-pulse responses, the set of records required to carry out the "double-pulse" subtraction procedure (Kiss \& Shizgal, 1989) was collected at each C-T interval. This set included: (1) records of the response to a pair of pulses with the $C-T$ interval set to a value within the range tested in the behavioral phase of the experiment $(0.15-5.0 \mathrm{msec})$, (2) records of the response to a single $C$ pulse synchronized with the $\mathrm{C}$ pulse in Record 1, (3) records of the response to a pair of pulses with the $C-T$ interval set to $0.4 \mathrm{msec}$ and the $T$ pulse synchronized with the $T$ pulse in Record 1, and (4) records of the response to a single $C$ pulse synchronized with the $C$ pulse in Record 3. At least eight traces were obtained in each condition so that averaging could be used to improve the signal-to-noise ratio. Thus, at least 32 traces were collected for each C-T interval tested.

At a C-T interval of $0.4 \mathrm{msec}$, we observed little sign of either local potential summation or recovery from refractoriness. Thus, subtracting Record 3 from Record 4 was expected to yield an essentially "pure" T-pulse artifact. Subtracting Record 2 from Record 1 was expected to yield a T-pulse artifact and a T-pulse response. Hence, subtracting the "pure" artifact from this latter record was expected to yield an isolated T-pulse response. The assumptions underlying this procedure, as well as its advantages and shortcomings, are discussed in detail by Kiss and Shizgal (1989).

\section{RESULTS}

\section{Stimulation Sites}

As shown in Figure 1, the tips of the LH stimulation electrodes were clustered in the perifornical area of the medial forebrain bundle, within a region extending 2.8-3.3 $\mathrm{mm}$ behind bregma, $1.2-1.9 \mathrm{~mm}$ lateral to the midline, and 8.3-9.7 $\mathrm{mm}$ below the surface of the skull (Paxinos \& Watson, 1986).

\section{Behavioral Data}

Refractory-period estimates. Figure 2 is an example of the curves yielded by Yeomans's (1975) procedure for

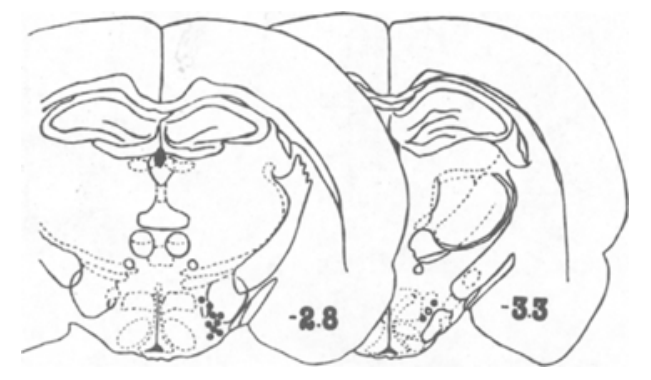

Figure 1. Location of the tips of the stimulation electrodes according to the Paxinos and Watson (1986) atlas. The number in the lower right-hand corner of each section indicates its distance (in $\mathrm{mm}$ ) behind bregma. 


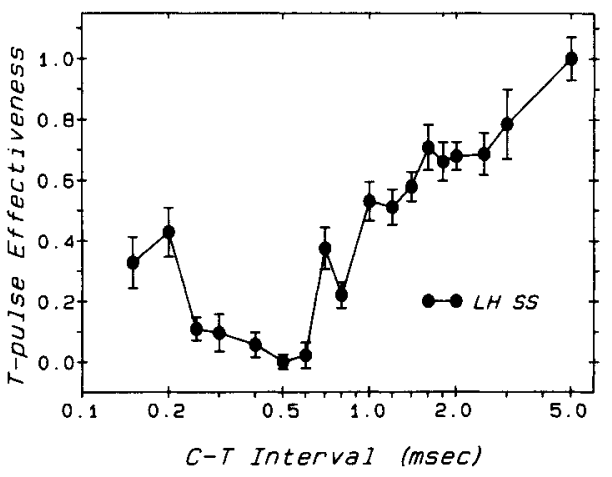

Figure 2. Effect of the $\mathrm{C}-\mathrm{T}$ interval on $\mathrm{T}$-pulse effectiveness in the behavioral test. The higher the $T$-pulse effectiveness, the fewer the number of pulse pairs required to sustain a half-maximal rate of responding. Error bars are the SEMs of five observations. estimating the refractory periods of the directly stimulated neurons responsible for the rewarding effect of brain stimulation. According to the simplest assumptions (Gallistel, Shizgal, \& Yeomans, 1981), such curves show how changing the $\mathrm{C}-\mathrm{T}$ interval alters the ratio of the number of reward neurons fired by the $C$ and $T$ pulses. At the longest $\mathrm{C}-\mathrm{T}$ intervals, all of the neurons fired by the $\mathrm{C}$ pulses will have recovered from refractoriness by the time the $T$ pulse is administered. Consequently, the T-pulse effectiveness statistic will equal 1 , indicating that the $T$ pulse fires as many reward neurons as the $\mathrm{C}$ pulse. When the $\mathrm{C}-\mathrm{T}$ interval is decreased sufficiently, some of the neurons will not yet have recovered sufficiently to respond to the $\mathrm{T}$ pulse. Thus, the $\mathrm{T}$ pulse will fire relatively fewer neurons, and the effectiveness value will decline. When the $\mathrm{C}-\mathrm{T}$ interval is reduced to very low values, all of the neurons fired by the $C$ pulse will be refractory to excita-

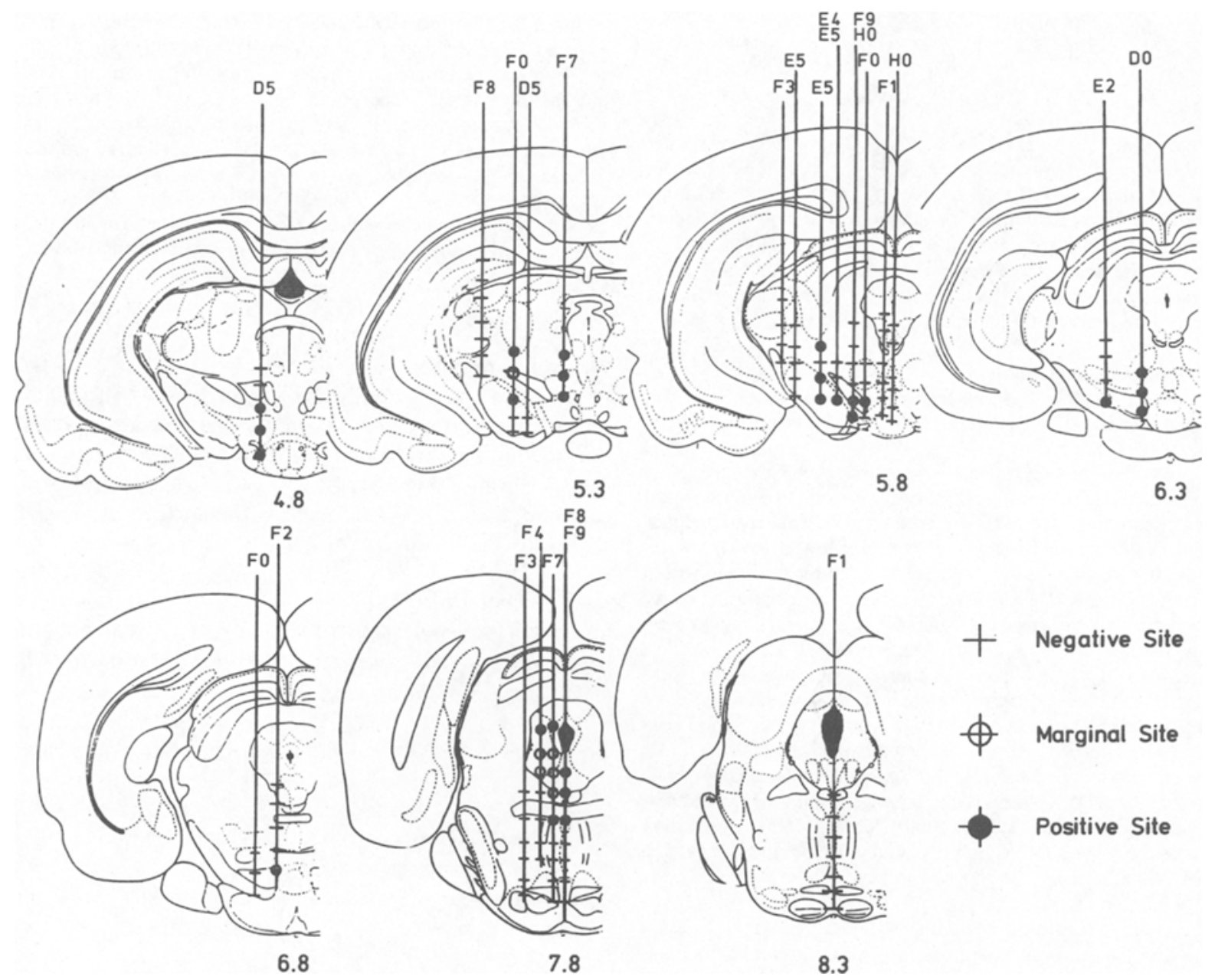

Figure 3. Location of recording sites. Filled circles = sites where directly driven compound action potentials were recorded, hatch marks = negative sites, and open circles = marginal sites. The number below each section indicates its distance (in mm) behind bregma. 
tion by the $\mathrm{T}$ pulse. However, the $\mathrm{T}$ pulse may fire neurons just beyond the region excited by the $C$ pulse as a result of the summation of subthreshold depolarizations ("local potential summation"). The shorter the C-T interval, the greater the summation of these rapidly decaying local potentials.

At most LH sites tested in previous studies, T-pulse effectiveness values decline from $0.2-0.4 \mathrm{msec}$, begin to rise when the C-T intervals exceeds $0.4-0.6 \mathrm{msec}$, complete most of their rise by $1.2-1.5 \mathrm{msec}$, and thereafter may climb more gradually (Shizgal \& Murray, 1989). The curves obtained from the 16 sites tested in the present study generally conform to this pattern. For example, the curve depicted in Figure 2 declines from $0.2-0.4 \mathrm{msec}$, begins to rise when the $\mathrm{C}-\mathrm{T}$ interval exceeds $0.7 \mathrm{msec}$, completes most of its rise by $1.6 \mathrm{msec}$, and rises more gradually from $2.5-5.0 \mathrm{msec}$. This curve deviates from the pattern generally observed only in that the mean $T$-pulse effectiveness value for the shortest $C$ - $T$ interval is lower than the value for the next shortest interval, a result that appears inconsistent with the notion that the early segment of the curve represents the decay of local potentials. Although the large error associated with these two measurements might suggest that this discrepancy is simply due to random variation, a similar pattern was seen at several other sites. It is not clear whether this pattern is due to a physiological process or to an unexpected fea-

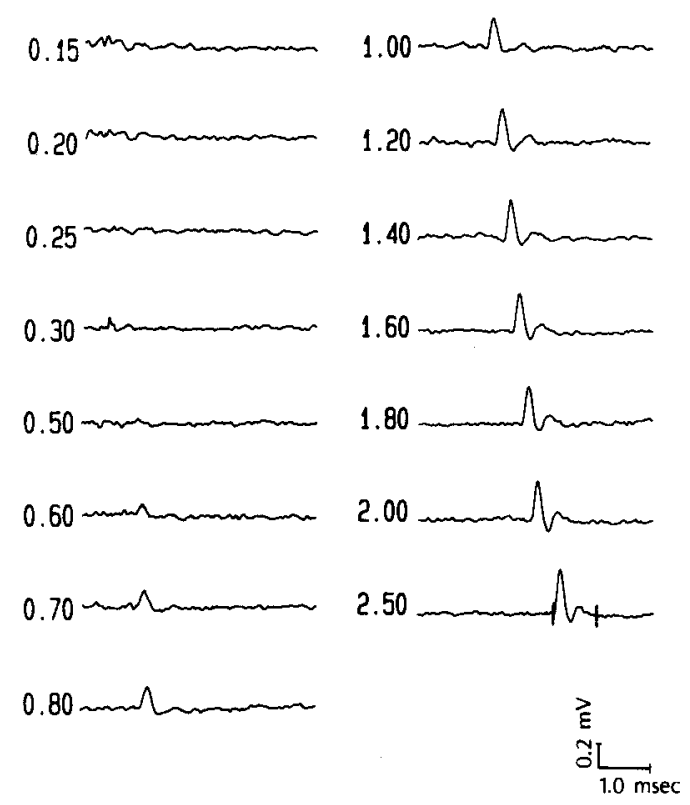

Figure 4. Recovery from refractoriness in the neurons responsible for a compound action potential recorded in the ventral tegmental area. The $C$-pulse artifact, $C$-pulse response, and much of the T-pulse artifact have been removed by means of digital subtraction. As described in the text, each of these subtracted records, as well as each record in Figures 5 and 6, was derived from four sets of averaged "raw" records. The numbers to the left of the traces are the C-T intervals (in msec).

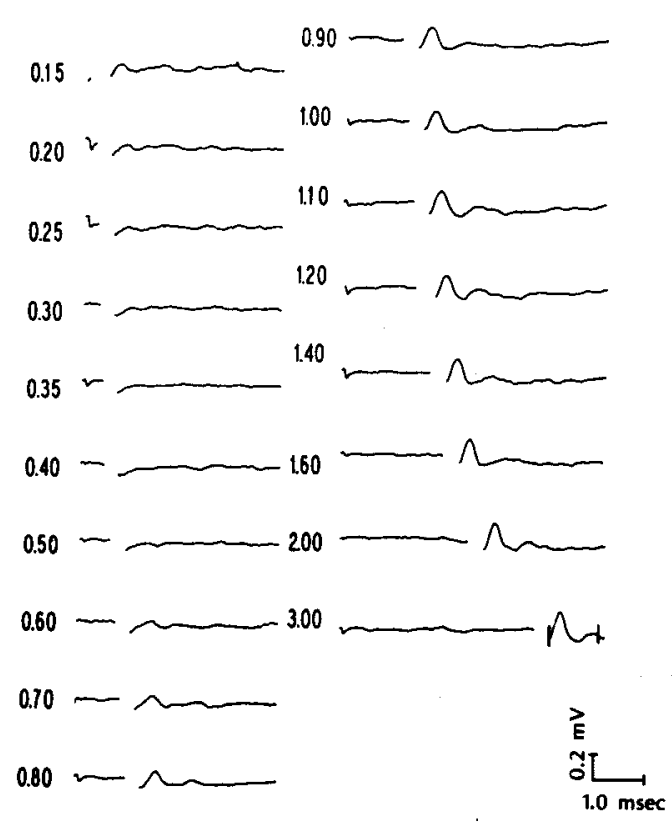

Figure 5. Recovery from refractoriness in the neurons responsible for a compound action potential recorded in the substantia nigra. The $C$-pulse artifact, $C$-pulse response, and much of the $T$-pulse artifact have been removed by means of digital subtraction; the gap in the traces is due to our erasure of a portion of the T-pulse artifact that remained following subtraction. The numbers of the left of the traces are the $\mathrm{C}-\mathrm{T}$ intervals (in msec).

ture of the apparatus such as an interaction in the outputgating circuitry of the stimulator.

\section{Electrophysiological Data}

Response criteria. Recording sites were classified as "positive" and the responses as directly driven CAPs when a signal was clearly separable from the background noise, the response latency was stable, and the response was not markedly attenuated by increasing the stimulation frequency to $100 \mathrm{~Hz}$.

Recording sites. As shown in Figure 3, positive sites (filled circles) were found over an anterior-posterior extent of 3.5 mm (from 4.8-8.3 mm behind bregma). Many of these sites were within $0.5 \mathrm{~mm}$ of the boundaries of the VTA, the dorsomedial portion of the SN, the ventromedial portion of the PAG, or the medial portion of the decussation of the superior cerebellar peduncle. In several cases, the transition from a positive to a negative site (hatch marks) or vice versa occurred as the electrode was lowered by $0.5 \mathrm{~mm}$. Marginal cases are denoted by open circles.

Refractory-period estimates. Figure 4 describes recovery from refractoriness in neurons responsible for CAPs recorded at a VTA site. To produce this set of traces, records obtained at different $\mathrm{C}$ - $\mathrm{T}$ intervals were processed by means of the double-pulse subtraction technique (Kiss \& Shizgal, 1989). If the technique worked perfectly, each trace would consist of an isolated T-pulse response. Given that the $\mathrm{C}$ pulse was delivered at the same location on 
each trace, each T-pulse response was displaced from its predecessor by the sum of the increment in the C-T inter$\mathrm{val}$ and any change in the latency of the T-pulse response. No clear sign of a response was evident at $0.5 \mathrm{msec}$, but a response began to emerge from the noise at a $\mathrm{C}-\mathrm{T}$ interval of $0.60 \mathrm{msec}$. This response grew steadily as the $\mathrm{C}-\mathrm{T}$ interval was further increased, and eventually stabilized. The small, early perturbations in the traces obtained at $\mathrm{C}$ - T intervals of $0.15,0.20$, and $0.30 \mathrm{msec}$ were probably remnants of the C-pulse artifact.

The subtracted traces in Figure 5 were derived from records obtained at a recording site in the $\mathrm{SN}$. The residual T-pulse artifacts, which were larger than in Figure 4, were erased for the sake of clarity. At C-T intervals of 0.15 and $0.20 \mathrm{msec}$, one can discern small $\mathrm{T}$-pulse responses that were probably due to local potential summation. As the $\mathrm{C}-\mathrm{T}$ int:rval was increased, the $\mathrm{T}$-pulse response initially decayed but reemerged at a C-T interval of $0.6 \mathrm{msec}$; the response grew as the $\mathrm{C}-\mathrm{T}$ interval was further increased, presumably due to recovery from refractoriness.

Figure 6 presents sets of T-pulse responses recorded at a site in the PAG. The signal-to-noise ratio was substantially lower than in Figures 4 and 5 , as was generally the case in responses recorded in this region. Nonetheless, a response did emerge eventually from the noise at a $\mathrm{C}-\mathrm{T}$ interval of $1.0 \mathrm{msec}$ and grew as the $\mathrm{C}-\mathrm{T}$ interval was increased.

Calculation of response area. As a means of quantifying the changes in the response, we elected to com-

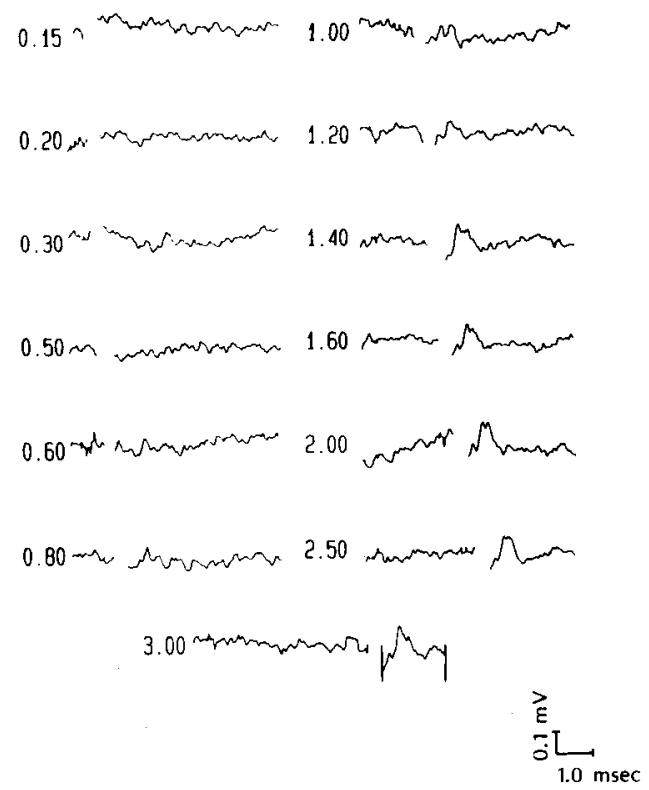

Figure 6. Recovery from refractoriness in the neurons responsible for a compound action potential recorded in the periaqueductal gray matter. The $C$-pulse artifact, $C$-pulse response, and much of the T-pulse artifact have been removed by means of digital subtraction; the gap in the traces is due to our erasure of a portion of the T-pulse artifact that remained following subtraction. The numbers to the left of the traces are the $\mathrm{C}-\mathrm{T}$ intervals (in msec). pute a measure related to the area of the response within a given time window. This was accomplished by summing the absolute value of the deviation of each point within the window from the mean value within the window. The mean was used in lieu of the baseline in order to remove the contribution of residual, long-duration components of the shock artifact that might have shifted the response along the voltage axis. The window was defined in terms of the response at the longest C-T interval tested; its leading edge was the point at which the response emerged from the baseline noise, and its trailing edge was the point at which the record returned to baseline at the end of the response. An example of such a window is the segment demarcated by two vertical lines in the bottom right-hand trace in Figure 4 (C-T interval $=2.5 \mathrm{msec}$ ). Each record in a given data set was evaluated within the same time window.

An estimate of the relative effectiveness of the $T$ pulse in driving CAPs was obtained by first finding the $\mathrm{C}-\mathrm{T}$ intervals at which the estimate of response area was smallest and largest, and then using these values to scale the set of area estimates according to the following formula:

$$
E_{\mathrm{CAP}_{\mathrm{CT}}}=\frac{\left(\text { Area }_{\mathrm{CT}}-\text { Area }_{\min }\right)}{\left(\text { Area }_{\max }-\text { Area }_{\min }\right)},
$$

where $E_{\mathrm{CAP}_{\mathrm{CT}}}=$ estimated effectiveness of the $\mathrm{T}$ pulse in driving CAPs at a given $\mathrm{C}-\mathrm{T}$ interval, Area $_{\mathrm{CT}}=$ area of the T-pulse response at a given C-T interval, Area ${ }_{\max }=$ maximum of the areas of all of the $T$-pulse responses, and Area $_{\min }=$ minimum of the areas of all of the T-pulse responses. This transformation forces the minimum $E$ value to zero and the maximum to 1 . Figure 7 shows the results of this calculation for the three sets of traces in Figures 4, 5 , and 6 . The underlying assumptions and limitations of the area measure are discussed below.

\section{Comparison of Behavioral and Electrophysiological Data}

To facilitate comparison of the behavioral and electrophysiological measures, the behavioral data were rescaled so that the $T$-pulse effectiveness values varied from zero to 1:

$$
E_{\mathrm{CT}}^{\prime}=\frac{\left(E_{\mathrm{CT}}-E_{\min }\right)}{\left(E_{\max }-E_{\min }\right)},
$$

where $E_{\mathrm{CT}}^{\prime}=$ T-pulse effectiveness at a given C-T interval rescaled to vary between zero and $1, E_{\mathrm{CT}}=\mathrm{T}$-pulse effectiveness at a given $\mathrm{C}-\mathrm{T}$ interval according to Equation $1, E_{\max }=$ maximum $\mathrm{T}$-pulse effectiveness according to Equation 1, and $E_{\min }=$ minimum T-pulse effectiveness according to Equation 1.

Figure 8 combines the rescaled behavioral data from Figure 2 with the VTA CAP data from Figures 4 and 7 . Note the substantial overlap in the range of C-T intervals over which the two curves rise. At the seven recording sites in and near the VTA, this overlapping region represents an average of $81 \%$ of the range of $\mathrm{C}-\mathrm{T}$ intervals over which the behavioral curves rise. 


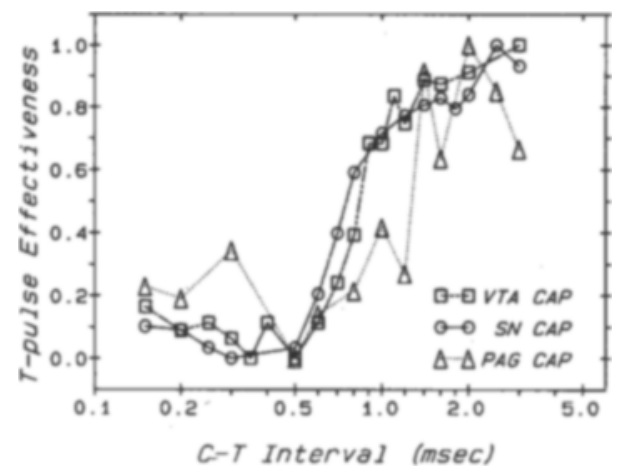

Figure 7. Effect of the $\mathrm{C}-\mathrm{T}$ interval on the relative effectiveness of the $\mathrm{T}$ pulse in driving compound action potentials (CAPs) recorded at sites in the ventral tegmental area (VTA), substantia nigra (SN), and periaqueductal gray matter (PAG). The T-pulse effectiveness measure expresses, as a proportion of the range of areas observed, the difference between the area of the $T$-pulse response at a given C-T interval and the minimum area observed. Thus, T-pulse effectiveness is forced to vary between 0 and 1.

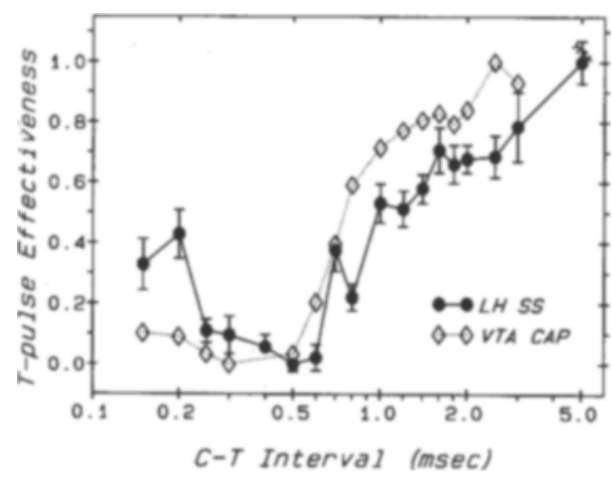

Figure 8. Comparison of a behaviorally derived measure of recovery from refractoriness to a measure derived from compound action potentials (CAPs) recorded in the ventral tegmental area (VTA). The filled circles represent the behavioral data from Figure 2, rescaled according to Equation 3 (SS = self-stimulation). The open diamonds represent the CAP data from Figure 4. The tip of the stimulating electrode was located at the following coordinates from the Paxinos and Watson (1986) atlas: $A P=$ bregma $-2.8, L=2.0$, and $V=-9.6$. The coordinates for the recording site were: $A P=$ bregma $-5.8, L=0.8$, and $V=-8.0$.

Figures 9 and 10 compare CAP data from sites in the SN (Figures 5 and 7) and PAG (Figures 6 and 7) to the corresponding behavioral data. Again, there is substantial overlap in the range of $\mathrm{C}-\mathrm{T}$ intervals over which the behaviorally and electrophysiologically derived curves rise. This overlap averaged $89 \%$ in the case of the results from the six SN sites and $87 \%$ in the case of the results from the eight PAG sites.

Figure 11 demonstrates variation in the electrophysiologically derived recovery curves as a function of the recording site; both sets of CAPs were driven by the same stimulation electrode, current, and pulse duration. The open triangles represent CAPs obtained at a site in the medial portion of the SN, whereas the inverted open triangles represent CAPs obtained at a second SN site, $0.4 \mathrm{~mm}$ lateral and $0.6 \mathrm{~mm}$ dorsal to the first site. The corresponding behavioral results are denoted by filled circles.

\section{DISCUSSION}

The results of this experiment confirm and extend the findings of our initial study (Shizgal et al., 1982). As predicted by the results of the behavioral collision experi-

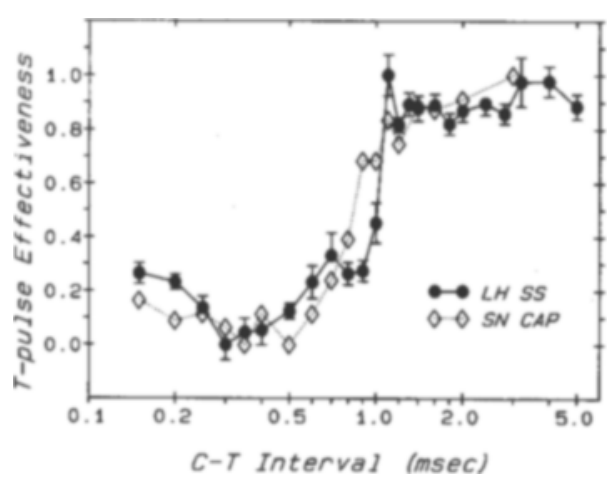

Figure 9. Comparison of a behoviorally derived measure of recovery from refractoriness to a measure derived from compound action potentials (CAPs) recorded in the substantia nigra (SN). The filled circles represent behavioral data rescaled according to Equation 3 (SS = self-stimulation); the open diamonds represent the CAP data from Figure 5 . The tip of the stimulating electrode was located at the following coordinates from the Paxinos and Watson (1996) atlas: $A P=$ bregma $-2.8, L=1.8$, and $V=-9.2$. The coordinates for the recording site were: $A P=$ bregma $-6.3, L=1.6$, and $V=-8.2$.

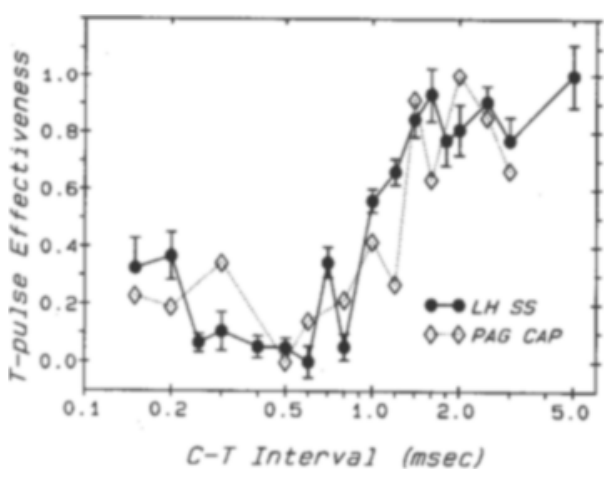

Figure 10. Comparison of a behaviorally derived measure of recovery from refractoriness to a measure derived from compound action potentials (CAPs) recorded in the periaqueductal gray matter (PAG). The filled circles represent behavioral data rescaled according to Equation 3 (SS = self-stimulation); the open diamonds represent the CAP data from Figure 6. The tip of the stimulating electrode was located at the following coordinates from the Paxinos and Watson (1986) atlas: $A P=$ bregma $-2.8, L=1.7$, and $V=-8.5$. The coordinates for the recording site were: $A P=b r e g m a-7.8, L=0.0$, and $V=-6.5$. 


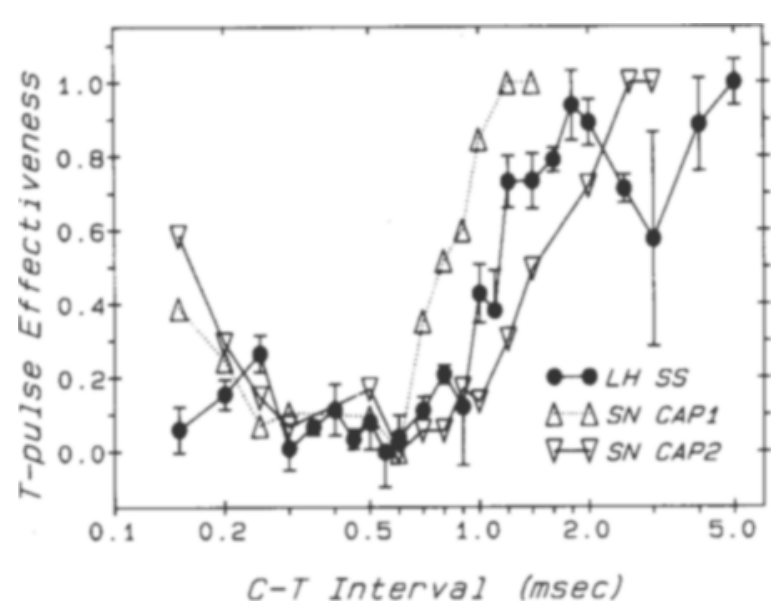

Figure 11. Comparison of recovery from refractoriness in the neurons responsible for compound action potentials (CAPs) recorded at two substantia nigra (SN) sites. The open triangles represent CAP data from a site in the medial SN (AP $=$ bregma $-5.8, L=1.5$, and $V=-8.6$ and the inverted open triangles represent CAP data from a second site $0.4 \mathrm{~mm}$ lateral and $0.6 \mathrm{~mm}$ dorsal to the first. The same stimulation electrode, current, and pulse duration were used to drive both sets of CAPs. The tip of the stimulating electrode was located at the following coordinates: $A P=$ bregma -2.8 , $L=1.9$, and $V=-9.3$.

ments (Bielajew \& Shizgal, 1982; Shizgal et al., 1980), rewarding stimulation of the LH elicited CAPs at recording sites in the VTA. The refractory periods of the neurons responsible for the rewarding effect overlap substantially with the refractory periods of the neurons responsible for the CAPs. This overlap was much easier to discern in the present study because of the improved response isolation achieved by means of the double-pulse subtraction technique. In addition to the responses observed at VTA sites, CAPs were recorded at sites in and near the SN and PAG that were not tested in our initial study. The electrophysiological measures of recovery from refractoriness obtained at these sites also overlap the behaviorally derived values.

Before discussing the implications of these findings, some of the assumptions underlying the comparison of the behavioral and electrophysiological measures must be examined. Most important are the assumptions pertaining to the measurement of response area and to the scaling of the effectiveness values.

\section{The Area Measure}

The T-pulse responses in Figures 4, 5, and 6 change in an orderly manner as a function of the C-T interval. In order to compare the CAP data to the behavioral results, it was necessary to compute a single measure to summarize these changes. We elected to use a measure related to response area rather than a measure of response amplitude because it was easier to apply such a measure in a consistent manner to CAPs differing substantially in form and because an area measure was expected to be more sensitive to the recruitment of small fibers. When the $\mathrm{C}-\mathrm{T}$ interval is just long enough to result in an ob- servable response, only the most excitable fibers will respond to the $\mathrm{T}$ pulse. At longer $\mathrm{C}-\mathrm{T}$ intervals, slower conducting fibers with longer refractory periods may also be recruited. If so, the CAP will spread out in time at long $\mathrm{C}-\mathrm{T}$ intervals as the action potentials propagating along the smaller, slower conducting fibers lag behind those propagating along the larger fibers. When this lag exceeds the duration of the more rapidly propagating action potentials, the peak amplitude of a response will no longer increase as a result of the recruitment of slower conducting fibers but the area of the response will continue to grow. Nonetheless, when the lag is less than the duration of the more rapidly propagating action potentials, an area measure might underestimate the contribution of the slower fibers because of the partial superimposition of opposite phases of the action potentials in the slower and faster fibers.

Despite its limitations, we believe the area measure to be adequate for the purposes of comparing the $\mathrm{C}-\mathrm{T}$ intervals over which the neurons responsible for the behavioral and electrophysiological data recover from refractoriness. The key point is that the bias we have mentioned should primarily affect the form of the recovery curve rather than the range over which it rises. Although the contributions of slowly recovering fibers might be underestimated, recruitment of such fibers should nonetheless increase response area. Thus, one can think of the bias as bending the recovery curve but not altering the points at which it begins to rise and approaches asymptote. If so, a comparison restricted to the ranges of the curves would appear to be valid.

\section{Effects of Rescaling}

Another point of concern is the possible effect of the scaling procedures on the form and range of the recovery curves. Recall that an $E$ value of zero was assigned to the $\mathrm{C}-\mathrm{T}$ interval at which the response with the smallest area was observed. This was done in order to minimize the contribution of background noise to the area measure. However, an $E$ value of zero implies that the T pulse failed to fire any of the neurons responsible for the CAPs. If the process of local potential summation has not yet run its course by the time recovery from refractoriness begins, the $T$ pulse will fire at least some neurons at all $\mathrm{C}-\mathrm{T}$ intervals. By forcing the curve to begin its rise from zero, we may be inadvertently overestimating the onset of recovery from refractoriness. This problem could be at least partially circumvented by using $\mathrm{T}$ pulses of greater amplitude than the $\mathrm{C}$ pulses, thus reducing the size of the region in which local potential summation can occur (Yeomans, Matthews, Hawkins, Bellman, \& Doppelt, 1979). An analogous argument holds for the behavioral data.

A second problem with the scaling procedures is the assignment of an $E$ value of 1 to the C-T interval at which the response with the largest area was observed. An $E$ value of 1 implies that the T pulse fired all of the neurons responsible for the $\mathrm{C}$-pulse response. However, it 
is possible that recovery was not always complete at the longest C-T interval tested (e.g., Figures 4 and 5). Had we tested longer intervals, we might have found that the recovery curves would have continued to climb. One solution to this problem is to use the single-pulse subtraction technique (Kiss \& Shizgal, 1989) to isolate the response to a single stimulation pulse and then to compare the areas of the T-pulse responses from each paired-pulse record to the area of this response.

\section{Sampling Bias}

In a related study, Rompré and Shizgal (1986) recorded the antidromic responses of single neurons in the septum and basal forebrain in response to stimulation of the $\mathrm{LH}$ and VTA. The refractory periods of $64 \%$ of these neurons were greater than $1.4 \mathrm{msec}$. In contrast, neurons with refractory periods longer than $1.4 \mathrm{msec}$ do not make pronounced contributions to many of the CAPs (but see "CAP2" in Figure 11). One possible explanation of this discrepancy is that the axons of many of the slowly recovering neurons reach the LH but not the VTA. Indeed, $73 \%$ of the cells driven from the LH, but only $48 \%$ of the cells driven from the VTA in Rompre and Shizgal's sample, had refractory periods longer than $1.4 \mathrm{msec}$. Another hypothesis is that the larger currents flowing during action potentials in larger, more rapidly recovering fibers tend to overshadow the weaker signals produced by smaller, more slowly recovering fibers.

\section{Implications of the Resemblance Between the Behavioral and Electrophysiological Results}

Given that the same electrodes, currents, and pulse durations were used in the behavioral and electrophysiological phases of this study, one can assume that the neurons responsible for the CAPs were a subset of the neurons directly activated during the self-stimulation testing. Notwithstanding the possibility of sampling bias and distortions introduced by the scaling procedures, there is remarkable agreement between the range of $\mathrm{C}-\mathrm{T}$ intervals over which the behaviorally and electrophysiologically derived curves rise. In the case of the VTA recording sites, such an overlap was predicted from the results of behavioral collision experiments. If, as the behavioral collision data suggest, reward-related neurons directly link the LH stimulation sites to the VTA, then the spectrum of fibers contributing to the CAPs must include at least part of the spectrum suggested by the behavioral estimates of recovery from refractoriness in reward-related neurons directly activated by LH stimulation.

In the results of a previous behavioral experiment (Macmillan, Simantirakis, \& Shizgal, 1985), there was considerable overlap between estimates of recovery from refractoriness in the neurons responsible for the rewarding effect of $\mathrm{LH}$ and $\mathrm{SN}$ stimulation. The results obtained at the $\mathrm{SN}$ recording sites in the present study suggest this resemblance may be more than coincidental: the CAP data show that rewarding stimulation of the $\mathrm{LH}$ activates neurons that project directly to the $\mathrm{SN}$ and that have refractory periods similar to those of the neurons responsible for the rewarding effect. Thus, the fibers linking the $\mathrm{LH}$ and $\mathrm{SN}$ may include the axons of reward-related neurons. The behavioral version of the collision technique offers a ready means of testing this hypothesis.

A subpopulation of descending MFB fibers have been shown to turn laterally in the general vicinity of the VTA and course through the pars compacta of the SN (Arbuthnott, Mitchell, Tulloch, \& Wright, 1976; Hosoya \& Matsushita, 1981; Nauta \& Domesick, 1982; Saper, Swanson, \& Cowan, 1979). The similarity of the behaviorally derived estimates of recovery from refractoriness at LH, VTA, and SN self-stimulation sites and the CAP data from the present study are consistent with the idea that reward-related neurons compose part of this projection. The notion that reward-related fibers activated by LH stimulation reach the SN via the VTA might be tested if one could first demonstrate collision between the SN and $\mathrm{LH}$ in the behavioral test and then determine whether small VTA lesions alter the magnitude of the collision effect or the magnitude of CAPs recorded in the SN.

The descending MFB fibers that course laterally through the pars compacta of the SN eventually turn dorsomedially as well as caudally, ultimately reaching the PAG. They are joined in the PAG by another group of descending MFB fibers that follow a more medial trajectory en route from the VTA (Arbuthnott et al., 1976; Hosoya \& Matsushita, 1981; Nauta \& Domesick, 1982; Saper et al., 1979). Thus, depending on the location of the recording site, either or both groups of fibers might be responsible for the CAPs recorded in the PAG following stimulation of the LH. The positive sites in the vicinity of the decussation of the superior cerebellar peduncle lie near the trajectory of the medial group of fibers.

Behaviorally derived estimates of the refractory periods of reward-related neurons directly activated by stimulation of the ventral portion of the PAG overlap with estimates obtained from LH self-stimulation sites (Bielajew, Jordon, Ferme-Enright, \& Shizgal, 1981; Rompre \& Miliaressis, 1987). Recently, Boye and Rompré (1987) used the behavioral version of the collision test to demonstrate that reward-related fibers directly link the VTA and PAG. By showing that fibers linking the LH to the PAG have refractory periods consistent with the estimates obtained for reward-related neurons, the results of the present study reinforce the implication of Boye and Rompre's study that it would be worthwhile to carry out behavioral collision tests between the LH and PAG. However, the poor signalto-noise ratios of the CAPs recorded in the PAG in the present study might suggest that relatively few stimulated fibers link the two sites and that such an experiment would thus be difficult to carry out.

\section{Future Applications}

Although similar recovery curves were obtained from widely separated recording sites, marked changes in the curves were sometimes observed as a result of relatively small displacements of the recording electrode. For ex- 
ample, the two electrophysiologically derived curves in Figure 11 rise over substantially different intervals but were recorded at sites separated by only $0.4 \mathrm{~mm}$ laterally and $0.6 \mathrm{~mm}$ ventrally. Both CAPs were driven by the same LH stimulation electrode, current, and pulse duration. Thus, the differences between them cannot be due to differences in the conditions of stimulation and must reflect differences in the trajectories of fibers that recover from refractoriness at different $\mathrm{C}-\mathrm{T}$ intervals. This result suggests that CAP recording might be used to map the trajectories of different fiber types activated at a single selfstimulation site. Carrying out such an experiment would appear particularly worthwhile in light of Gratton and Wise's (1985) demonstration that different portions of behaviorally derived refractory-period curves obtained from self-stimulating rats can be dissociated pharmacologically.

The reselts of the present study illustrate the complementarity of CAP recording and behavioral methods for inferring physiological characteristics of neurons responsible for effects of electrical brain stimulation. For example, by revealing points along the trajectories of fibers activated by rewarding stimulation and describing the recovery from refractoriness in these fibers, the CAP data suggest new sites for future collision tests. In carrying out such collision studies, it would be wise to record stimulationelicited CAPs during the positioning of the stimulation electrodes in order to ensure an optimal alignment. In addition, CAP recording might be used to assess the conclusions of behavioral collision experiments that were carried out in the absence of concurrent electrophysiological measurements. Although anatomical studies can determine whether fibers directly link pairs of sites at which collision-like effects have been reported, CAP recording can determine whether such fibers are actually stimulated at the parameters used in the behavioral experiments.

\section{REFERENCES}

Arbuthnott, G. W., Mitchell, M. J., Tulloch, I. F., \& WRIGHT, A. K. (1976). Efferent pathways from lateral hypothalamic neurones. Journal of Physiology (London), 263, 131-132.

Bielajew, C., Jordon, C., Ferme-Enright, J., \& Shizgal, P. (1981). Refractory periods and anatomical linkage of the substrates for lateral hypothalamic and periaqueductal gray self-stimulation. Physiology \& Behavior, 27, 95-104.

Bielajew, C., \& Shizgal, P. (1982). Behaviorally derived measures of conduction velocity in the substrate for rewarding medial forebrain bundle stimulation. Brain Research, 237, 107-119.

Boye, S., \& RoMPrÉ, P.-P. (1987). Evidence for a direct axonal link between reward-relevant neurons in the ventral tegmental area-posterior hypothalamus and the medial mesencephalon. Society for Neuroscience Abstracts, 13, 1544.
Gallistel, C. R., Shizgal, P., \& Yeomans, J. S. (1981). A portrait of the substrate for self-stimulation. Psychological Review, 88, 228-273.

Gratton, A., Wise, R. A. (1985). Hypothalamic reward mechanism: Two first-stage fiber populations with a cholinergic component. Science, 227, 545-548.

Hosoya, Y., \& MATsushita, M. (1981). Brainstem projections from the lateral hypothalamic area in the rat, as studied with autoradiography. Neuroscience Letters, 24, 111-116.

KISS, I., \& SHIzGAL, P. (1989). Improved artifact rejection and isolation of compound action potentials by means of digital subtraction. Journal of Neuroscience Methods, 30, 219-229.

Macmillan, C. J., Simantirakis, P., \& Sizgal, P. (1985). Selfstimulation of the lateral hypothalamus and ventrolateral tegmentum: Excitability characteristics of the directly stimulated substrates. Physiology \& Behavior, 35, 711-723.

Mund, W. (1980). A constant-current stimulator. Physiology \& Behavior, 24, 991-993.

NAutA, W. J. H., Domesick, V. B. (1982). Neural associations of the limbic system. In A. L. Beckman (Ed.), The neural basis of behavior (pp. 175-206). New York: Spectrum.

Paxinos, G., Watson, C. (1986). The rat brain in stereotaxic coordinates (2nd ed.). New York: Academic Press.

Rompré, P.-P., Miliaressis, E. (1987). Behavioral determination of refractory periods of the brainstem substrates of self-stimulation. Behavioral Brain Research, 23, 205-219.

RomprÉ, P.-P., SHIzGAL, P. (1986). Electrophysiological characteristics of neurons in forebrain regions implicated in self-stimulation of the medial forebrain bundle in the rat. Brain Research, 364, 338-349.

Saper, C. B., Swanson, L. W., Cowan, W. M. (1979). An autoradiographic study of the efferent connections of the lateral hypothalamic area in the rat. Joumal of Comparative Neurology, 183, 689-706.

Shizgal, P., Bielnew, C., Corbett, D., Skelton, R., \& Yeomans, J. S. (1980). Behavioral methods for inferring anatomical linkage between rewarding brain stimulation sites. Journal of Comparative \& Physiological Psychology, 94, 227-237.

Shizgal, P., Bielajew, C., Kiss, I. (1982). Psychophysical and electrophysiological studies of the substrate for brain stimulation reward. In B. G. Hoebel \& D. Novin (Eds.), The neural basis of feeding and reward (pp. 419-429). Brunswick, ME: Haer Institute.

Shizgal, P., \& MURRaY, B. (1989). Neuronal basis of intracranial selfstimulation. In J. M. Liebman \& S. J. Cooper (Eds.), The neuropharmacological basis of reward (pp. 106-163). Oxford: Oxford University Press.

YeOMANS, J. S. (1975). Quantitative measurement of neural poststimulation excitability with behavioral methods. Physiology \& Behavior, 15, 593-602.

Yeomans, J. S., Matthews, G. G., Hawkins, R. D., Bellman, K., \& Doppelt, H. (1979). Characterization of self-stimulation neurons by their local potential summation properties. Physiology \& Behavior, 22, 921-929.

Yeomans, J. S., Mercouris, N., \& Ellard, C. (1985). Behaviorally measured refractory periods are lengthened by reducing electrode tip exposure or raising current. Behavioral Neuroscience, 99, 913-928.

(Manuscript received July 31, 1989; revision accepted for publication November 17, 1989.) 